\title{
FIXAÇÃO DE CATETERES VENOSOS PERIFÉRICOS EM CRIANÇAS: ESTUDO COMPARATIVO
}

\author{
Setting of peripheral venous catheters in children: comparative study \\ Fijación de catéteres venosos periféricos en niños: estudio comparativo
}

Luis Manuel Cunha Batalha'

Luísa Paula Santos Costa

Dulce Maria Gomes de Almeida ${ }^{3}$

Patrícia Adriana Almeida Lourenço ${ }^{4}$

Amélia Maria Ferreira Maia Gonçalves ${ }^{5}$

Ana Cristina Guerra Teixeira ${ }^{6}$

\section{RESUMO}

0 estudo descreve e compara dois métodos de fixação de Cateteres Venosos Periféricos, com e sem uso de imobilização com tala, sua interferência no conforto, desenvolvimento das atividades de vida e complicações clínicas, e seu tempo de permanência. Tratase de um estudo prospectivo e descritivo que envolve crianças internadas em dois hospitais, com idades até 10 anos e que necessitaram de colocação de cateteres venosos periféricos, com punção com agulha tipo abocath. A seleção dos casos foi de natureza consecutiva. A análise de 59 casos revela que a fixação dos cateteres venosos periféricos com uso de tala interfere nas atividades de vida, mas reduz os riscos de aparecimento de complicações. 0 uso desta técnica permanece controverso, e o seu uso rotineiro requerer uma decisão racional, dada a sua interferência nas atividades de vida da criança.

Palavras-chave: Cateterismo Venoso Periférico. Imobilização. Criança.

\begin{abstract}
The present study describes and compares two methods for inserting Peripheral Venous Catheters with and without the use of a splint, and their impact on comfort, performance of daily activities, clinical complications and duration of application. This is a prospective and descriptive study involving children aged up to 10 years hospitalized in two hospitals. They required the insertion of Peripheral Venous Catheters and were punctured using an abocath needle. Patients were selected on a consecutive basis. The analysis of 59 cases shows that the insertion of Peripheral Venous Catheters with the use of a splint interferes in Daily Activities but reduces the risk of complications. The use of this technique is still controversial and its routine application requires a rational decision due to its impact on the child's daily activities.
\end{abstract}

Keywords: Catheterization, Peripheral. Immobilization. Child.

\begin{abstract}
Resumen
El estudio describe y compara dos métodos de fijación de catéteres venosos periféricos con y sin recurso a inmovilización con férula, y su interferencia en la comodidad, desarrollo de actividades del día a día, complicaciones clínicas y su persistencia. Se realizó un estudio prospectivo y descriptivo que se aplicó a niños hasta los 10 años ingresados en dos hospitales y que necesitaban catéteres venosos periféricos recurriendo a punción con una aguja tipo abocath. La selección de los 59 casos que fueron objeto de este estudio fue de naturaleza consecutiva. El análisis llevado a cabo ha demostrado que la fijación de catéteres venosos periféricos con recurso a una férula interfiere en las actividades del día a día pero disminuye el riesgo de posibles complicaciones. Esta técnica sigue siendo polémica y su uso rutinario requiere una decisión ponderada, dada su interferencia en las actividades de la vida del niño.
\end{abstract}

Palabras claves: Cateterismo Periférico. Inmovilización. Niño

\footnotetext{
${ }^{1}$ Professor Adjunto da Unidade Científico-Pedagógica da Escola Superior de Enfermagem de Coimbra, Coimbra-Distrito de Coimbra-Portugal. E-mail: batalha@esenfc.pt, ¿Licenciada em Enfermagem. Enfermeira Especialista em Saúde Infantil e Pediátrica do Departamento Pediátrico do Centro Hospitalar de Coimbra, EPE,Coimbra-Distrito de Coimbra- Portugal. E-mail:luisapaulasantoscosta@gmail.com, ${ }^{3}$ Licenciada em Enfermagem. Enfermeira Especialista em Saúde Infantil e Pediátrica do Departamento Pediátrico do Centro Hospitalar de Coimbra, EPE, Coimbra-Distrito de Coimbra-Portugal. Email:dulcealmeida@gmail.com, ${ }^{4}$ Licenciada em Enfermagem. Enfermeira Especialista em Saúde Infantil e Pediátrica do Departamento Pediátrico do Centro Hospitalar de Coimbra, EPE, Coimbra-Distrito de Coimbra- Portugal.patricialourenco@msn.com, ${ }^{5}$ Licenciada em Enfermagem. Enfermeira Graduada do Serviço de Lactentes do Centro Hospitalar do Porto, EPE - Hospital Maria Pia,Coimbra-Distrito de Coimbra- Portugal. E-mail:ameliamaia@gmail.com, ${ }^{6}$ Mestre em Ciências de Enfermagem. Enfermeira Chefe do Serviço de Lactentes do Centro Hospitalar do Porto, EPE - Hospital Maria Pia, Porto-Distrito de Porto-Portugal. E-mail:acris.guerra@clix.pt
} 
Fixação de Cateteres venosos periféricos em crianças

\section{INTRODUÇÃO}

A introdução de Cateter Venoso Periférico (CVP) é um dos procedimentos mais frequentemente realizados por enfermeiros em pediatria. ${ }^{1}$ Este ato terapêutico está indicado nas situações em que se requer um acesso venoso para a administração de fluidos, fármacos ou obtenção de sangue.

Para a sua realização, o enfermeiro necessita de habilidade e conhecimento que lhe permitam, em função das finalidades terapêuticas, material de punção disponível e condições da rede venosa da criança, determinar o local de punção e o método de fixação do cateter mais adequado para prevenir a ocorrência de possíveis complicações como flebites, infiltração/extravasamento ou interferências na qualidade de vida das crianças, confor to e atividades de vida (AV).

0 local de punção para colocação de CVP na criança, sem prejuízo de outros fatores, depende das características anatomofisiológicas relacionadas com a sua idade. Os locais de eleição são as veias superficiais dos membros superiores, preferencialmente os locais mais distantes e o membro não dominante ou cuja mão não seja usada pela criança. ${ }^{1-3}$ As veias do couro cabeludo podem ser uma alternativa em recém-nascidos (RN) ou mesmo lactentes até os 9 meses por facilidade de acesso ${ }^{2}$, e as veias do pé podem ser utilizadas nas crianças que ainda não adquiriram a capacidade de gatinhar ou andar. ${ }^{2}$

0 material de punção habitualmente usado em crianças são os cateteres flexíveis tipo abocath com mandril metálico. Em pediatria, ao contrário do tratamento em adultos, estes cateteres devem ser mantidos até que sejam observados sinais de complicações, ${ }^{1}$ e os cuidados a ter no local da punção parecem não ter qualquer associação com a ocorrência de complicações. ${ }^{4} 0$ uso de uma compressa estéril sobre o local da punção e o uso de uma película transparente ou simplesmente de uma fita adesiva não são consensuais. ${ }^{5-7}$

A técnica de fixação dos CVP com uso de imobilização com tala para restrição ou limitação dos movimentos articulares próximo do local da punção está indicado nas mãos, punhos, cotovelo e maléolo. ${ }^{2,3,6} \mathrm{~A}$ recomendação é para se utilizar uma tala acolchoada, para proteger as regiões de maior atrito, fixada com fita adesiva e mantida de modo a não prejudicar a observação do local de inserção ou limitar a circulação. ${ }^{2,3,8}$ No entanto, o uso de imobilização com tala apresenta-se como uma prática controversa. Em um estudo realizado em crianças até os 12 anos utilizando imobilização com tala, em mais de metade dos casos (55,3\%) ocorreu infiltração, o que indicia a ineficácia desta prática. ${ }^{9}$

Os estudos sobre as técnicas de fixação dos CVP são escassos, e as práticas baseiam-se na maioria dos casos em comportamentos ritualistas sem evidência científica ${ }^{7}$. A imobilização pode oferecer proteção e segurança, mas pouco se sabe da sua interferência na ocorrência de possíveis complicações como flebites, infiltração/extravasamento, interferências nas AV e desconforto da criança. Por outro lado, o uso desta prática parece não se coadunar com 0 atual uso de cateteres flexíveis tipo abocath em detrimento de agulhas metálicas. Talvez por isso comecem a surgir novas técnicas de fixação dos CVP que não exigem imobilização das articulações com talas.

Ao verificarmos práticas de enfermagem diferentes em dois hospitais pediátricos portugueses sobre a forma de fixação dos CVP em crianças (com uso e sem uso de imobilização), pretendemos descrever e comparar estes dois métodos de fixação de CVP quanto à sua interferência no conforto e desenvolvimento das AV da criança, complicações clínicas e tempo de permanência dos cateteres.

\section{METOLOGIA}

Estudo prospectivo, descritivo e controlado cujo objetivo foi comparar dois métodos de fixação de CVP (envolvendo ou não a imobilização com tala do local adjacente à inserção do cateter), relativamente à sua interferência no confor to e desenvolvimento das AV da criança, complicações clínicas e tempo de permanência dos cateteres.

Estes dois métodos de fixação dos CVP diferiam entre dois hospitais pediátricos portugueses quanto ao uso ou não de imobilização com tala rígida. Em um, é pratica habitual o uso dessa imobilização para manter 0 CVP; no outro, não se usa qualquer tipo de imobilização. Para além desta particularidade, a técnica de fixação do CVP é semelhante em ambos os hospitais, consistindo em colocar três pequenas tiras de adesivo, duas para fixar o cateter, sendo uma em gravata, e uma terceira para fixar o cateter e o prolongador da perfusão. A área é coberta com circulares de adesivo ou é usada uma película transparente estéril sobre o local da punção. Para melhor fixação, em um dos hospitais coloca-se uma pequena compressa e protege-se tudo com surgifix (malha tubular elástica, confeccionada com fios de algodão, poliéster e látex). No outro hospital, a área é imobilizada com a aplicação de uma tala no lado oposto à colocação do cateter, caso este seja aplicado no dorso da mão, antebraço ou pé. Esta tala tem como objetivo a imobilização da articulação do local da punção e é fixa com duas tiras de adesivo nas extremidades.

A colheita de dados decorreu durante um ano entre os meses de novembro de 2008 e novembro de 2009 no Centro Hospitalar do Porto, EPE / Hospital 
Maria Pia, serviço de lactentes e no Centro Hospitalar de Coimbra, EPE / Departamento Pediátrico, serviços de cirurgia/queimados, ortopedia/neurocirurgia e urgência. Participaram no estudo crianças internadas com idades até 4 anos (no Departamento Pediátrico do Centro Hospitalar de Coimbra, EPE, foi aumentada a idade de recrutamento até 10 anos) e que necessitaram de colocação de CVP para soroterapia, antibioterapia ou outro tratamento, sempre por via intravenosa contínua, qualquer que fosse o diagnóstico, puncionados com uma agulha tipo abocath. A seleção dos casos foi de natureza consecutiva em função do dia, turno e disponibilidade dos enfermeiros que colaboraram na colheita dos dados.

Os dados foram colhidos por um enfermeiro em cada serviço com recurso a uma folha de registo integrada em cada processo clínico da criança seleccionada, sem a sua identificação. As folhas receberam números para controle e foram recolhidas no momento da alta. 0 estudo envolveu, direta e indiretamente, as crianças baseando-se a recolha dos dados na observação e descrição de acontecimentos, tais como a data e hora de colocação de CVP, data e hora de remoção do mesmo, acrescido da informação do motivo. Neste estudo, foram apenas considerados os dados relativos ao primeiro CVP colocado à criança. No momento da remoção definitiva do CVP e antes da alta, os pais que acompanharam mais de $50 \%$ do tempo de internamento da criança foram questionados para se saber até que ponto a criança se sentiu desconfor tável ou impedida de realizar as suas atividades de vida diária. Estas mesmas questões foram também colocadas às crianças com idade igual ou superior a 4 anos. Os enfermeiros que colheram os dados não interferiram na técnica de fixação dos CVP, descrevendo somente o que observaram, e entrevistaram o enfermeiro que executou a técnica para saber o fundamento da sua intervenção. Por vezes, foi necessário consultar o processo clínico para completar a recolha dos dados.

A análise estatística foi realizada com o auxílio do programa SPSS $®$ - Statistical Package for the Social Sciences versão 15.0 para o Windows $®$ (Statistical Product and Service Solutions, Inc., Chicago, II, EUA). 0 ajustamento à Normal das variáveis foi verificado por meio do teste Shapiro-Wilk, não assumindo nenhuma variável. 0 estudo descritivo dos dados foi feito para as variáveis categóricas pelas frequências absolutas e relativas percentuais e para as variáveis contínuas através dos quartis e limites mínimo e máximo. As diferenças entre as técnicas de fixação dos CVP foram analisadas pelo Teste Qui-quadrado para variáveis categóricas e pelo teste de Mann-Whitney U para variáveis intervalares. Para correlacionar tempo de permanência do CVP em ambas as técnicas de fixação do CVP e a idade da criança com o permanência do CVP, foi usado o teste de correlação de Spearman. As diferenças foram consideradas estatisticamente significativas sempre que $p<0,05$. Os dados obtidos por entrevista a pais, criança ou enfermeiros foram analisados de acordo com o conteúdo, seguindo a proposta de Bardin ${ }^{9}$ para identificação de palavras-chave.

Esta investigação teve o parecer favorável dos responsáveis pelos serviços, departamento, conselhos de administração e comissão de ética de ambos os centros hospitalares e foi realizada de acordo com a Declaração de Helsínquia da Associação Médica Mundial. Aos participantes foi dado documento informativo do estudo juntamente com declaração de consentimento informado e garantia de proteção da identidade e do anonimato dos dados.

\section{RESULTADOS}

Participaram no estudo 59 crianças com idades até 10 anos, das quais pelo menos $75 \%$ tinham idade inferior a 4 anos. 0 gênero masculino representou $52,5 \%$ da população estudada, sendo esta distribuída quase equitativamente entre 0 Centro Hospitalar do Porto, EPE / Hospital Maria Pia (CHP) e o Centro Hospitalar de Coimbra, EPE / Departamento Pediátrico (CHC). As 29 (49,2\%) crianças do CHP encontravam-se internadas no serviço de lactentes, e, no CHC, a maioria encontrava-se no serviço de cirurgia/queimados (17; $28,8 \%)$.

A maioria das crianças estava internada por patologia do foro médico $(35 ; 59,3 \%)$. Ao longo do internamento foram colocados na cada criança um a cinco CVP, sendo que em pelo menos metade houve apenas necessidade de colocar um só CVP.

0 local escolhido para colocar o CVP foi a mão $51(86,4 \%)$, com uma distribuição entre o lado direito e esquerdo semelhante, $26(44,1 \%)$ e 33 $(55,9 \%)$, respectivamente.

Das 30 (50,8\%) fixações dos CVP com tala de imobilização, o motivo dessa técnica foi, segundo o enfermeiro que a executou, o risco de remoção em $24(40,7 \%)$ dos casos.

0 tempo de permanência dos CVP variou entre 1 e 202 horas, com uma mediana de 72 horas. 0 principal motivo para a remoção do CVP foi a indicação médica $(34 ; 57,6 \%)$, embora em 19 $(32,2 \%)$ dos casos fosse a infiltração/extravasamento (Quadro I). 
Fixação de Cateteres venosos periféricos em crianças

Quadro I-Dados demográficos e clínicos

\begin{tabular}{|c|c|}
\hline \multicolumn{2}{|l|}{ Dados demográficos e clínicos $(n=59)$} \\
\hline Idade (anos) $\left[\mathbf{P}_{25}, \mathbf{P}_{50}, \mathbf{P}_{75}\right.$ (mín-máx)] & $0,0,4(0-10)$ \\
\hline Sexo masculino [número (\%)] & $31(52,5)$ \\
\hline \multicolumn{2}{|l|}{ Hospital } \\
\hline CHP-EPE, Hospital Maria Pia & $29(49,2)$ \\
\hline CHC-EPE, Departamento Pediátrico & $30(50,8)$ \\
\hline \multicolumn{2}{|l|}{ Serviço } \\
\hline Lactentes (CHP) & $29(49,2)$ \\
\hline Cirurgia/queimados (CHC) & $17(28,8)$ \\
\hline Urgência (CHC) & $7(11,9)$ \\
\hline Ortopedia / neurocirurgia (CHC) & $6(10,2)$ \\
\hline \multicolumn{2}{|l|}{ Diagnóstico [número (\%)] } \\
\hline Médico & $35(59,3)$ \\
\hline Cirúrgico & $24(40,7)$ \\
\hline Número de cateteres por criança $\left[\mathbf{P}_{25}, \mathbf{P}_{50}, \mathbf{P}_{75}\right.$ (mín-máx)] & $1,1,2(1-5)$ \\
\hline \multicolumn{2}{|l|}{ Local de inserção } \\
\hline Mão & $51(86,4)$ \\
\hline Pé & $4(6,8)$ \\
\hline Punho & $2(3,4)$ \\
\hline Antebraço & $1(1,7)$ \\
\hline Crânio & $1(1,7)$ \\
\hline \multicolumn{2}{|l|}{ Lado de inserção } \\
\hline Direito & $26(44,1)$ \\
\hline Esquerdo & $33(55,9)$ \\
\hline \multicolumn{2}{|l|}{ Técnica de fixação } \\
\hline Com imobilização & $30(50,8)$ \\
\hline Sem imobilização & $29(49,2)$ \\
\hline \multicolumn{2}{|l|}{ Motivo de imobilização } \\
\hline Risco de remoção & $24(40,7)$ \\
\hline Baixa idade & $2(3,4)$ \\
\hline Alteração da consciência & $1(1,7)$ \\
\hline Sem justificação & $3(5,1)$ \\
\hline $\begin{array}{l}\text { Tempo de permanência do CVP (horas) }\left[\mathbf{P}_{25}, \mathbf{P}_{50}, \mathbf{P}_{75} \text { (mín- }\right. \\
\text { máx)] }\end{array}$ & $48,72,120(1-202)$ \\
\hline \multicolumn{2}{|l|}{ Motivo de remoção } \\
\hline Quando indicado & $34(57,6)$ \\
\hline Infiltração/extravasamento & $19(32,2)$ \\
\hline Obstrução & $2(3,4)$ \\
\hline Remoção acidental & $2(3,4)$ \\
\hline Flebite & $1(1,7)$ \\
\hline Outro & $1(1,7)$ \\
\hline
\end{tabular}

Ao analisarmos o motivo de remoção do CVP segundo a extravasamento (12 versus 7), dois únicos casos de obstrução técnica de fixação, verificamos que, quando não se usou a tala e um único caso de flebite e mau funcionamento do cateter de imobilização, surgiram mais casos de infiltração/ (Gráficol).

Gráfico I - Motivo de remoção do CVP segundo a técnica de fixação

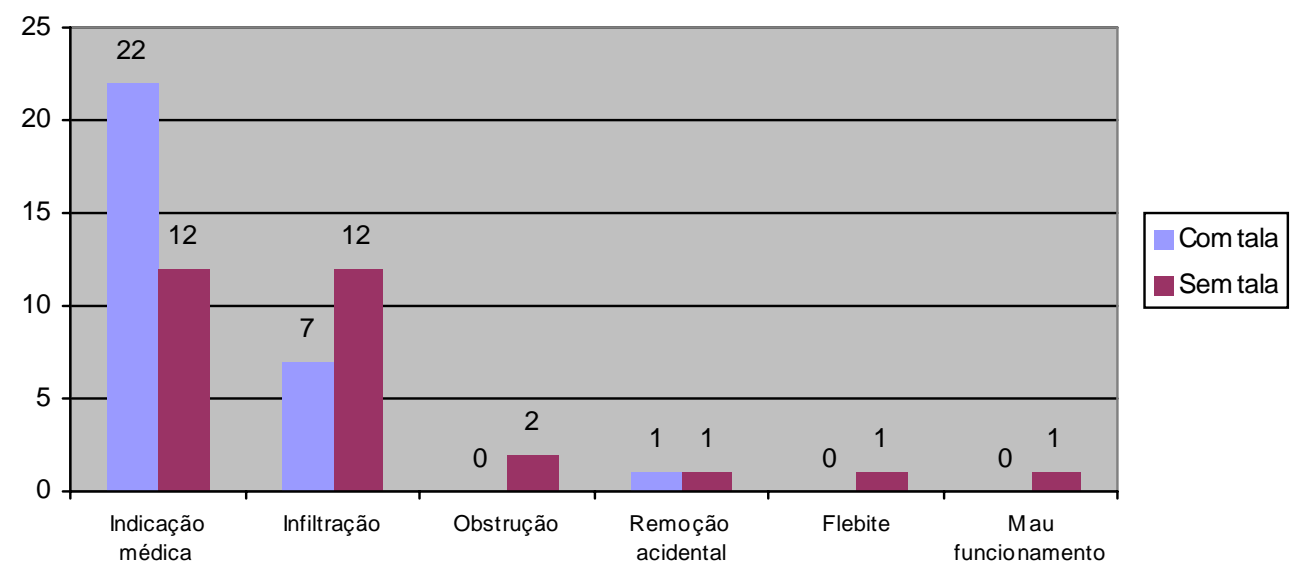


A opinião da maioria das mães foi a de que a criança não sentiu desconforto com a presença do CVP (43; 72,9\%). Das que referiram algum tipo de desconforto mencionam como causa principalmente a imobilidade (4;6,8\%) e o não brincar $(3 ; 5,1 \%)$.
Houve interferência nas $\mathrm{AV}$, segundo as mães, em cerca de $23(39,0 \%)$ crianças com relevo, para as AV: divertir-se, $12(20,3 \%)$, e comer/beber e vestir-se, com $6(10,1 \%)$, ambas (Quadro II).

Quadro II - Opinião das mães sobre a interferência da técnica de fixação no conforto e AV.

\begin{tabular}{|lc|}
\hline \multicolumn{2}{|c|}{ Opinião das mães sobre o conforto e interferência nas AV } \\
\hline Desconforto & $16(27,1)$ \\
Sim & $43(72,9)$ \\
Não & \\
Tipo de desconforto & $4(6,8)$ \\
Imobilidade & $3(5,1)$ \\
Não brinca & $2(3,4)$ \\
Inflamação & $1(1,7)$ \\
Alimentação & $1(1,7)$ \\
Vestir & $1(1,7)$ \\
Dormir & $3(5,1)$ \\
Outras & $1(1,7)$ \\
Desconhecido & \\
Interferência nas atividades de vida & $23(39,0)$ \\
Sim & $36(61,0)$ \\
Não & \\
Tipo de interferência nas atividades de vida & $12(20,3)$ \\
Divertir-se & $6(10,1)$ \\
Vestir-se & $6(10,1)$ \\
Comer/beber & $1(1,7)$ \\
Higiene pessoal & $1(1,7)$ \\
Eliminar & $1(1,7)$ \\
Mobilização & $1(1,7)$ \\
Dormir & $1(1,7)$ \\
Todas as AV & \\
\hline
\end{tabular}

As 14 crianças com idade superior a 4 anos encontraramse divididas $(11,9 \%)$ quanto ao conforto ou desconforto sentido pela presença de imobilização com tala. Das que manifestam desconforto, a maioria $(4 ; 6,8 \%)$ referiu o fato de ter a mão presa.
Quanto à interferência nas suas AV, 10 (16,9\%) crianças fizeram essa alusão, sendo as AV divertir-se, 5 $(8,5 \%)$, e o comer/beber, 4 (6,8\%), as mais prejudicadas (Quadro III).

Quadro III - Opinião das crianças sobre a interferência da técnica de fixação no seu conforto e AV.

\begin{tabular}{|lc|}
\hline \multicolumn{2}{|c|}{ Opinião das crianças sobre conforto e interferência nas AV } \\
\hline Desconforto & $7(11,9)$ \\
Sim & $7(11,9)$ \\
Não & \\
Tipo de desconforto & $4(6,8)$ \\
Mão presa & $1(1,7)$ \\
Mão presa e prurido & $1(1,7)$ \\
Não brinca & $1(1,7)$ \\
Desconhecido & $10(16,9)$ \\
Interferência nas atividades de vida & $4(6,8)$ \\
Sim & \\
Não & $5(8,5)$ \\
Tipo de interferência nas AV & $4(6,8)$ \\
Divertir-se & $2(3,4)$ \\
Comer/beber & $1(1,7)$ \\
Higiene pessoal & $1(1,7)$ \\
Mobilização & $1(1,7)$ \\
Dormir & \\
Todas as AV & \\
\hline
\end{tabular}


0 motivo de remoção do CVP devido a uma complicação, ou seja por não indicação médica, foi maior nos casos em que não se usou imobilização com tala $(p=0,018)$.

A escolha do local de inserção do CVP revelou ser independente da técnica usada $(p=0,959)$, o mesmo sucedendo para a opinião das mães quanto à interferência no conforto da criança $(p=0,613)$.

A técnica de fixação do CVP com imobilização com tala revelou, segundo opinião das mães, uma maior interferência nas AV da criança cuja diferença se revelou estatisticamente significativa ( $p=0,022)$.

0 tempo de permanência dos CVP foi semelhante em ambas as técnicas de fixação dos CVP $(p=0,976)$. No entanto, a correlação entre a idade e o tempo de permanência dos CVP em função da técnica de fixação revelou que, quanto maior é a idade da criança, menor é o tempo de permanência do CVP. Esta correlação revelou ser estatisticamente significativa quando não se usa imobilização com tala $(p=0,028)$ (Quadro IV).

Quadro IV - Relação entre o motivo de remoção, local de inserção, interferência no conforto e AV, e tempo de permanência do CVP com a técnica de fixação

\begin{tabular}{|c|c|c|c|c|}
\hline & \multicolumn{4}{|c|}{ Técnica de fixação } \\
\hline & Total & $\begin{array}{c}\text { Com } \\
\text { imobilização }\end{array}$ & $\begin{array}{c}\text { Sem } \\
\text { imobilização }\end{array}$ & $p$ \\
\hline Motivo de remoção & & & & $0,018^{*}$ \\
\hline Indicação médica & 34 & 22 & 12 & \\
\hline Complicação & 25 & 8 & 17 & \\
\hline Local de inserção do CVP & & & & $0,959^{\circ}$ \\
\hline Mão & 51 & 26 & 25 & \\
\hline Outro & 8 & 4 & 4 & \\
\hline Inter ferência no confor to (opinião da mãe) & & & & $0,613^{*}$ \\
\hline Sim & 16 & 9 & 7 & \\
\hline Não & 43 & 21 & 22 & \\
\hline Inter ferência nas AV (opinião da mãe) & & & & $0,022^{\circ}$ \\
\hline Sim & 23 & 16 & 7 & \\
\hline Não & 36 & 14 & 22 & \\
\hline $\begin{array}{l}\text { Tempo de permanência do CVP (horas) }\left[\mathrm{P}_{50}\right. \\
\text { (mín-máx)] }\end{array}$ & $\begin{array}{l}72(1- \\
202)\end{array}$ & $75,5(2-202)$ & $72(1-198)$ & $0,97 \sigma^{*}$ \\
\hline Idade - tempo de permanência do CVP (p)] & $\begin{array}{l}-0,155 \\
(0,240) \\
\end{array}$ & $\begin{array}{l}-0,188 \\
(0,319)^{\ngtr}\end{array}$ & $\begin{array}{c}-0,407 \\
(0,028)^{*}\end{array}$ & \\
\hline
\end{tabular}

* Teste do Qui-quadrado; ${ }^{\ddagger}$ Mann-Whitney U.; ${ }^{\ddagger}$ Coeficiente de Spearman.

\section{DISCUSSÃO}

Das 59 crianças que participaram no estudo, cerca de $75 \%$ tinham idade inferior a 4 anos e pelo menos metade eram lactentes (idade inferior a um ano). A comparação de idades entre os dois hospitais revela que no CHP todas as crianças tinham idade inferior a 4 anos, enquanto no $\mathrm{CHC}$ eram apenas 16 crianças. Este fato não pode ser esquecido na interpretação dos resultados.

A escolha do local para colocação do CVP foi quase sempre a mão, como se recomenda. ${ }^{2,5,10}$ Apesar de outros locais poderem ser utilizados, principalmente em lactentes (pés ou couro cabeludo), 2,3 essa não foi uma opção. A escolha do membro não dominante ou cuja mão não seja usada pela criança é um cuidado adicional a que o enfermeiro deve prestar atenção. ${ }^{1-3}$ Majoritariamente, os enfermeiros escolheram o lado esquerdo, o que indicia o cuidado que o enfermeiro tem quanto à influência deste particular aspecto no conforto e realização das AV da criança. Muito embora exista consenso de que a preferência por um dos lados começa a ficar evidente e definida na criança por volta dos 2,5 a 4 anos de idade, ${ }^{11}$ pensamos que esta particularidade merece ainda mais atenção, se atendermos a que cerca de $90 \%$ das pessoas são destras.

0 principal motivo referido pelos enfermeiros que usaram a técnica de fixação do CVP adicionando a imobilização com tala foi o risco de remoção acidental. Na verdade, em crianças, essa é uma possibilidade real ligada às suas naturais características do desenvolvimento (incompreensão e não aceitação voluntária de restrições, pouca noção de perigo, necessidade de atividade e recreação, e menor coordenação motora). Este aspecto ganha relevância se o CVP é colocado junto a uma articulação. Todavia, ao ser escolhido o dorso da mão, fora da articulação do punho e dedos, e usada uma agulha flexível tipo abocath, o risco de remoção diminui, como o parece comprovar este estudo com apenas dois casos de remoção 
acidental e sem relação com o uso da imobilização com tala. Outros estudos reforçam esta ideia da não eficácia da imobilização para diminuir o risco de remoção acidental do CVP do interior do vaso e consequente infiltração/ extravasamento. ${ }^{12}$

Em mais de metade dos casos, o motivo de remoção do CVP foi por indicação médica, o que demonstra a adequação dos cuidados prestados inerentes à sua manutenção. A prática de não substituição dos CVP até observação de sinais clínicos de complicações, ao contrário do que acontece em adultos, ${ }^{1,5}$ parece confirmar-se neste estudo em que mais de $75 \%$ permaneceram por mais de 5 dias. A principal causa de remoção do CVP, quando não prescrito, foi a infiltração/extravasamento ocorrendo em quase uma em cada três crianças.

Quando comparamos o motivo de remoção dos CVP (indicação médica e ocorrência de complicações) segundo a técnica de fixação dos CVP, verificamos que quando não se usou a imobilização com tala houve mais nove casos de complicações com os CVP (infiltrações/extravasamento, obstruções, flebites e mau funcionamento). Os motivos para a ocorrência de complicações em CVP são várias, com evidência para infiltração/ extravasamento. Esta ocorrência pode ter múltiplas explicações, como a inadequada inserção do cateter, inadequada técnica asséptica, não deteç̧ão de quebras na integridade dos cateteres, fixação ineficaz, desajustado calibre e tipo de cateter, má condição das veias da criança, tipo de medicação e até má filtração dos fluidos. ${ }^{12}$ No entanto, o não uso de imobilização com tala parece ser um outro fator associado ao aparecimento de mais complicações.

Independentemente da técnica de fixação dos CVP, a idade da criança correlacionou-se negativamente com o seu tempo de permanência. No entanto, quando não se usou a tala de imobilização esta associação revelou-se estatisticamente significativa. Ou seja, quanto maior a idade da criança menor é o tempo de permanência dos CVP. Uma vez que neste grupo as idades das crianças são menores (todas abaixo dos 4 anos), esta variável pode interferir de forma diferenciada na condição clínica da criança, na adequação do calibre do cateter, na diluição, no volume e na velocidade da perfusão, de forma a enviesar os resultados, pelo que se recomenda um novo estudo com um controle mais efetivo da variável idade nos dois grupos. Esta constitui, aliás, a principal limitação deste estudo.

Pouco mais de $25 \%$ das mães revelou que o seu filho sentiu algum desconforto com a presença do CVP, com evidência para a sensação de imobilidade e de não poder brincar. A interferência nas AV aconteceu, segundo as mães, em mais de $1 / 3$ dos casos, sendo as atividades divertir-se, vestir-se e comer/ beber as mais afectadas.

Segundo opinião das mães, a fixação do CVP com uso de imobilização com tala interfere na realização de algumas AV do seu filho, mas não no seu conforto. No entanto, quando questionamos as 14 crianças que tinham idades acima dos 4 anos, sobre o confor to e interferência nas suas AV decorrentes da técnica de imobilização com tala, metade referiu sentir desconforto, sendo a mão presa a principal razão, e a maioria relatou interferência na realização das suas AV (divertir-se, vestir-se e comer/beber, as mesmas áreas identificadas pelas mães).

Um tempo mediano de 72 horas de permanência dos CVP está um pouco acima do encontrado em outros estudos. ${ }^{13-}$ ${ }^{15}$ Entre as duas técnicas de fixação dos CVP, não se encontram diferenças significativas, apesar de ligeiramente superior quando se usa imobilização com tala. As características anatomofisiopatologicas e psicomotoras inerentes à idade da criança podem influir no tempo de permanência dos CVP pelas suas diferenças em termos de patologias, suas necessidades terapêuticas, técnicas envolvidas e capacidade cognitiva e motora da criança. A tendência encontrada neste estudo é para que o tempo de permanência dos CVP diminua em função do aumento da idade. Todavia, esta associação é apenas evidente quando não se utiliza imobilização com tala, o que parece comprovar que, nestas circunstancias, quanto maior é a idade da criança menor é o tempo de permanência dos CVP. Assim sendo, a fixação dos CVP com uso de imobilização com tala parece não se justificar em crianças muito pequenas (lactentes).

A perda de informação pela não coincidência entre os turnos de trabalho do enfermeiro que recolheu os dados na admissão e na alta da criança limitou a dimensão da amostra. Por outro lado, o número reduzido de crianças com menos de 4 anos internadas em um dos hospitais dificultou a comparação dos grupos em função da idade. Sem prejuízo da replicação do estudo com um controle desta variável mais efetivo, os resultados parecem evidenciar que o uso da imobilização com tala para fixação dos CVP em crianças é vantajoso na prevenção de complicações e com reflexos no aumento do seu tempo de permanência, mas interfere nas AV da criança. Esta prática não deixa de ser controversa e exige uma decisão racional em função do contexto em que a criança se encontra e deve deixar de ser uma prática rotineira principalmente nas crianças mais pequenas.

\section{CONCLUSÕES}

Os resultados deste estudo parecem demonstrar que os enfermeiros escolhem preferencialmente a mão para colocar os CVP nas crianças, mas mais atenção deve ser dispensada quanto ao lado a escolher. Os que usam habitualmente a imobilização com tala para fixar os CVP justificam essa ação com o risco de remoção acidental, o que não parece ser confirmado neste trabalho (apenas ocorreram dois casos de remoção acidental). A maioria dos (VP foi removido por indicação médica embora as situações de infiltração / extravasamento não sejam de menosprezar. 
A fixação dos CVP com uso de imobilização com tala interfere nas $A V$ da criança e reduz os riscos de aparecimento de complicações, mas não interfere no tempo de permanência dos CVP. Em crianças abaixo dos 4 anos, a menor idade parece estar associada a um aumento do tempo de permanência do CVP quando não se usa a imobilização com tala, pelo que é uma prática a recomendar neste grupo etário.

A controvérsia do uso de imobilização com tala na fixação dos CVP em crianças permanece. No entanto, o seu uso rotineiro requerer uma decisão racional, dada a sua interferência nas AV da criança e de não oferecer proteção adicional para a manutenção dos cateteres em crianças mais pequenas.

Esperamos que este estudo contribua para o desenvolvimento de uma discussão mais aprofundada na área da técnica de fixação dos CVP e ajude a estimular a pesquisa nesta área de intervenção do enfermeiro.

\section{REFERÊNCIAS}

1. Centers for Disease Control and Prevention. Guidelines for the prevention of intravascular catheter- related infections. MMWR 2002; 51(RR-10).

2. Hockenberry MJ, Wilson D, Winkelstein ML. Fundamentos de Enfermagem Pediátrica Wong. Tradução de Alexandre Vianna Aldighieri Soares. $7^{\mathrm{a}}$ ed. São Paulo(SP): Elsevier; 2006.

3. Almeida FA, SabatésAL. Enfermagem pediátrica: a criança, o adolescente e a sua família no hospital. São Paulo(SP): Manole; 2008.

4. Hoffmann KK, Weber DJ, Samsa GP, Rutala WA. Transparent polyurethane film as an intravenous catheter dressing: a meta-analysis of the infection risks. JAMA 2002; 267: 2072-76.

5. Société Française d'Hygiène Hospitalière Prévention des infections liées aux cathéters veineux périphériques. [periódico o-line] Paris; 2005. Disponivel em: http://www.has-sante.fr/portail/upload/docs/application/ $\mathrm{pdf} /$ Catheters veineux 2005 fiche.pdf.

6. Machado AF, Pedreira MLG, Chaud MN. Estudo prospectivo, randomizado e controlado sobre o tempo de permanência de cateteres venosos periféricos em crianças, segundo três tipos de curativos. Rev Latino-am Enfermagem 2005; 13(3): 291-98.

7. Frey AM, Schears GJ. Why are we stuck on tape and suture? J Infus Nurs 2006; 29 (1): 34-8.

8. Machado AF, Pedreira MLG, Chaud MN. Eventos adversos relacionados ao uso de cateteres intravenosos periféricos em crianças de acordo com tipos de curativos. Rev Latino-am Enfermagem [periódico o-line]. 2008; 16 (3). Disponível em:http://www.scielo.br/scielo.php?pid=S010411692008000300005 \&script=sci arttext\&tlng=pt .

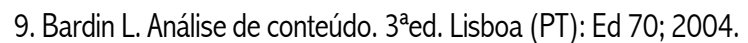

10. Bowden VR, Greenberg CS. Procedimentos de enfermagem pediátrica. Rio de Janeiro(RJ): Guanabara Koogan; 2005.
11. Martin WLB, Homci VPB, Silveira FM. 0 desenvolvimento de dominância manual e podálica em crianças destras e canhotas entre três a oito anos de idade. Interação Psicol 2009; 13(1): 37-47.

12. Phillips LD. Complicações da terapia intravenosa. In: Phillips LD. Manual de terapia intravenosa. $2^{\mathrm{a}}$ ed. Porto Alegre (RS): Artmed; 2001. p. 236-68.

13. Foster $L$, Wallis M, Paterson B, James $H$. A descriptive study of peripheral intravenous catheters in patients admitted to a pediatric unit in one australian hospital. J Infus Nurs 2002; 25(3):159-67.

14. Almonte R, Patole S, Muller R, Whitehall J. Comparison of two methods of taping peripheral intravenous cannulas. Indian Pediatr 1999; 36(5): 494-98.

15. Shimandle RB, Johnson D, Baker M, et al. Safety of peripheral intravenous catheters in children. Infect Control Hosp Epidemiol 1999; 20(11): 736-40.

\section{NOTAS}

Regina Paula Moita Esteves. Licenciada em Enfermagem. Enfermeira Especialista em Saúde Infantil e Pediátrica do Departamento Pediátrico do Centro Hospitalar de Coimbra, EPE,Coimbra-Distrito de Coimbra-Portugal. E-mail:rereginaesteves@gmail.com 